\title{
O DESEMPENHO DO HEDGE PARA CONTRATOS FUTUROS DE BOI GORDO: UMA ANÁLISE A PARTIR DAS PRINCIPAIS PRAÇAS PRODUTORAS BRASILEIRAS
}

\author{
Hedge Performance of the Cattle Futures Contract in Brazil
}

\begin{abstract}
RESUMO
O objetivo deste estudo se deu em analisar o desempenho do hedge de boi gordo na Brasil, Bolsa, Balcão (B3) considerando as principais praças produtoras do Brasil, avaliando a razão ótima e a efetividade do hedge. Para isso, aplicou-se uma derivação do modelo clássico de razão de hedge estática, incluindo um modelo de correção de erros (VEC) e, posteriormente, com a inclusão do modelo multivariado diagonal BEKK para análise da razão e efetividade do hedge dinâmicos. Considerando o período de análise ente 2012 a 2016, nota-se, em geral, uma baixa efetividade do hedge, sobretudo nas praças mais distantes da formação dos preços. O modelo dinâmico também sugere que em nenhum momento a razão ótima do hedge supera $50 \%$, indicando que o contrato futuro local não tem sido eficiente para atender à demanda de potenciais hedgers.
\end{abstract}

Luiz Eduardo Gaio

Universidade Estadual de Campinas

luizgaio@unicamp.br

Daniel Henrique Dario Capitani

Universidade Estadual de Campinas

danieldc@unicamp.br

Recebido em: 28/06/2018. Aprovado em: 11/03/2020.

Avaliado pelo sistema double blind review

Avaliador científico: Elisa Reis Guimarães

DOI: $10.48142 / 2238-68902019 v 21 n 1-3 p 6578$

\begin{abstract}
This paper examines the hedging performance of cattle futures contract at Brasil, Bolsa Balcão (B3), considering the main Brazilian livestock productions areas and assessing the different optimal hedge ratio and hedge effectiveness. Therefore, this study applied a derivation of the classical hedge ratio model, including the vector error corretion model (VECM), as well as the employment of the multivariated diagonal BEKK model for the analysis of dynamical hedge ratio and hedge effectiveness. Research dataset consist from 2012 through 2016. Overall, results point out to low hedge ratios, especially in the most distant production areas. In addition, the dynamic model also suggest that the optimal hedge ratio is lesser than $50 \%$, suggesting a weak efficiency provided by local cattle futures contract.
\end{abstract}

Palavras-chave: Derivativos Agropecuários. Efetividade do Hedge. Razão de Hedge.

Keywords: Agricultural Derivatives. Hedging Effectiveness. Hedge Ratio.

\section{INTRODUÇÃO}

A pecuária bovina e seu complexo constituem-se em um dos principais segmentos do agronegócio brasileiro, sendo o país o segundo maior produtor de carne bovina e o maior exportador. A atividade produtora está amplamente presente em diferentes regiões do país, concentrando-se, principalmente, nas regiões Norte, Centro-Oeste e Sudeste, destacando-se os estados de Mato Grosso, Minas Gerais, Goiás, Mato Grosso do Sul, Pará, Rondônia e Rio Grande do Sul (IBGE, 2015).

Sobre seu complexo, nota-se uma crescente concentração do abate de animais e comercialização de carnes, através de grandes grupos produtores, os quais cada vez mais interligados com outros elos da produção (URSO; BARRIONUEVO FILHO, 2008; MOITA; GOLON, 2014).
Em relação à produção, como bem destacam Monteiro et al. (2010), o risco de preços se inicia durante o momento de cria e engorda, em que não se pode prever o custo final de produção e, sobretudo, o preço de venda no momento de abate do animal. Isto se dá, principalmente, pela alta variação nos preços dos insumos e preços da commodity durante o período de engorda, além das incertezas acerca dos das oscilações nos mercados domésticos e externos, risco de doenças e barreiras sanitárias, e variação nos preços de bens substitutos.

De forma a reduzir os riscos de preços, os agentes atuantes neste setor produtivo têm a opção de estabelecimento de relações contratuais, como as operações com contratos futuros e de opções na bolsa. O contrato futuro de boi gordo na Brasil, Bolsa, Balcão (B3) tem se 
consolidado como um de seus principais contratos agrícolas, sobretudo a partir da segunda metade da década de 2000, com alto volume de negociação e montante transacionados.

Neste sentido, o presente trabalho tem como objetivo analisar a eficiência da operação do contrato futuro de boi gordo na B3 para redução do risco de oscilações do preço da commodity em diferentes praças comercializadoras, situadas nas principais regiões produtoras e abatedoras. Para isso, propõe-se, primeiramente, a estimação do ótimo que um agente comercializador de cada região deve hedgear no mercado futuro de forma a atingir a melhor relação risco-retorno. Além da razão de hedge ótima para o período de análise, pretende-se estimar uma razão ótima dinâmica no tempo, que permita identificar a variação da posição ótima do hedge em diferentes momentos do seu ciclo produtivo. Posteriormente, serão calculadas as efetividades do hedge para cada região.

Em geral, esta pesquisa se propõe a oferecer subsídios complementares para a análise da efetividade de hedge no mercado de futuros no Brasil e, desta maneira, permitir avaliar a efetividade do contrato vigente de boi gordo da B3 para hedgers de diferentes praças produtoras de gado.

\section{FUNDAMENTAÇÃO TEÓRICA E HIPÓTESES DO ESTUDO}

\subsection{Razão de Hedge de Mínima Variância}

Como amplamente discutido na literatura, o risco de base é resultado da diferença existente entre os preços correntes nos mercados físico e de futuros, tal como a posição assumida pelos hedgers. Assim, seu entendimento ou previsão tornam-se fatores de fundamental importância para o hedger, principalmente para que este assuma suas posições nos diferentes mercados (FLIGEWSKI, 1984; NETZ, 1996; LORD; TURNER, 1998). Desta forma, segundo Silveira (2003), os agentes devem definir uma proporção de suas posições à vista que irão assumir no mercado futuro, minimizando, assim, os riscos inerentes às variações nos preços de sua carteira. Tal proporção é conhecida como razão de hedge de mínima variância.

A decisão em relação a qual proporção das posições à vista será coberta por posições no mercado futuro é considerada por Myers e Thompson (1989) como um dos principais problemas enfrentados por traders de commodities agrícolas. Johnson (1960) deu início à formalização de uma teoria que buscasse determinar a proporção ideal da produção no mercado à vista a ser hedgeada no mercado futuro, modernizando a teoria do portfólio e adaptando-a ao problema do hedging. Ederington (1979), por sua vez, aprimorou um método para minimização do risco de preço, enquanto Hayenga e Di Pietre (1982) estimaram a razão de hedge ótima e a efetividade do hedge para atacadistas de produtos de origem suína e bovina que utilizavam tais respectivos contratos futuros.

De acordo com Myers e Thompson (1989), para equacionar-se a razão de hedge ótima, deve-se assumir que um agente tem a expectativa de negociar $N_{A}$ unidades de um ativo em um período. Tal como reforçado por Silveira (2003), para que o agente fixe seu preço de venda ou de compra, deve, obviamente, transacionar contratos futuros de seu ativo na ordem de $N_{F}$ unidades. Assim, a razão de hedege escolhida é expressa conforme equação 1 .

$h=\frac{N_{F}}{N_{A}}$

Aprimorando tal discussão, Cruz Júnior (2009) menciona que o modelo tradicional pode ser formulado a partir do problema de um produtor que opta realizar o hedge no mercado futuro, vendendo $N_{F}$ contratos a um preço $F_{l}$ no instante $t_{l}$. Ainda, supõe-se que a receita do produtor no mercado à vista seja conhecida somente no instante $t_{2}$, com a venda de $N_{S}$ unidades do produto a um preço $S_{2}$. Considerando que em $t_{2}$ o produtor inverta sua posição, comprando $N_{F}$ contratos a um preço $F_{2}$, as receitas nos mercados à vista e futuro, no momento $t_{2}$ serão estimadas conforme equações 2 e 3 .

$R_{S}=N_{S} S_{2}$

$R_{F}=N_{F}\left(F_{1}-F_{2}\right)=-N_{F}\left(F_{2}-F_{1}\right)$

Desta maneira, a receita total é dada pela soma de $R_{F}$ e $R_{S}$, tal como:

$R=N_{S} S_{2}-N_{F}\left(F_{2}-F_{1}\right)$

Substituindo (1) em (4), pode-se incorporar a razão de hedge na fórmula da receita, e então, encontrar a variância de $R$ que será minimizada. O problema da minimização da variância será obtido a partir da derivação deste termo em relação à razão de hedge $(h)^{1}$. Balizando-se no exemplo do produtor, de acordo com Hull (1995), este procura minimizar a variância de sua carteira (long no mercado à vista e short no mercado futuro) de modo a minimizar seus riscos.

${ }^{1} \mathrm{O}$ detalhamento algébrico desta formulação está expresso em Myers e Thompson (1989), Hull (1995, p. 113) e Silveira (2003). 
Tal formulação decorre do trabalho de Johnson (1960), que deriva a razão de hedge minimizando o risco do portfólio do hedger, assumindo que tal risco é dado pela variância das mudanças no valor do portfólio hedgeado, onde a minimização da variância de sua carteira resultará na razão de hedge ótima conforme equação 5 .

$h_{J}^{*}=\rho_{\Delta S, \Delta F} \frac{\sigma_{s}}{\sigma_{f}}=\frac{\operatorname{cov}\left(\Delta S_{t}, \Delta F_{t}\right)}{\operatorname{Var}\left(\Delta F_{t}\right)}$

sendo $\rho_{\Delta S, \Delta S} ; \sigma_{s}$ e $\sigma_{f}$ os desvios dos preços à vista e futuros, respectivamente; $\operatorname{Cov}\left(\Delta S_{t}, \Delta F_{t}\right)$ a covariância entre as diferenças dos preços à vista e futuros, e $\operatorname{Var}\left(\Delta F_{t}\right) \mathrm{e}$ $\operatorname{Var}\left(\Delta S_{t}\right)$ a variância da diferença dos preços futuros e físicos, respectivamente.

Diversos métodos foram desenvolvidos para a estimação da razão de hedge ótima, visando encontrar o grau ótimo de efetividade do hedge, como ressaltam Chen, Lee e Shrestha (2003). O modelo tradicional proposto por Johnson (1960) é baseado em uma regressão pelo método de mínimos quadrados ordinários (MQO), que considera mudanças nos preços à vista em virtude de variações nos preços futuros, onde a razão de hedge de mínima variância é encontrada pelo intercepto da equação, ou seja, é igual à reta que melhor se ajusta à regressão de mudança dos preços à vista contra mudanças nos preços futuros (CHEN, LEE, SHRESTHA, 2003; CRUZ JÚNIOR, 2009).

Segundo Marshall (1989, apud SILVEIRA, 2003), a efetividade do hedge depende de quanto à variação do preço à vista é compensada pela variação do preço futuro, dada a estimação da regressão, ou seja, depende do risco de base. Assim, obtém-se o grau de efetividade do hedge mediante o coeficiente de determinação da regressão $\left(R^{2}\right)$, o qual representa a correlação entre os preços à vista e futuros. Neste sentido, quanto maior for o $R^{2}$, maior a eficiência da operação, com tendência de menor risco de base.

Contudo, de acordo com Chen, Lee e Shrestha (2003), para que esta técnica seja válida e eficiente, algumas pressuposições associadas ao método de MQO devem ser satisfeitas. Um dos casos citados pelos autores é o problema da heterocedasticia no termo de erros da regressão. Também, como bem discutiu Myers e Thompson (1989), o parâmetro de deslocamento desta regressão estimada fornece apenas a razão oriunda da covariância incondicional entre as variáveis dependentes e explicativas para a variância incondicional das variáveis explicativas. Desta maneira, sugere-se o uso de informações de mercado relevantes e condicionadas à regressão, o que gerará covariâncias e variâncias condicionais, para que assim, seja analisado se esta abordagem é apropriada para a estimação da razão de hedge ótima.

Cruz Júnior (2009) discorre sobre a proposta de Myers e Thompson (1989) para a generalização do modelo tradicional. Tal generalização deve ser feita a partir de uma melhor especificação, utilizando-se ferramentais disponíveis na metodologia de séries temporais, de forma a evitar estimações equivocadas. Neste sentido, Viswanath (1993) complementa as considerações de Myers e Thompson (1989), considerando a dependência das convergências possíveis dos preços físicos e futuros e da razão de hedge em relação tanto à duração do hedge e quanto ao tempo de necessário para que o contrato atinja sua maturidade. Assim, ao invés de incluir um amplo número de defasagens na estimação da regressão, o autor recomenda a inclusão da base no modelo de regressão, como forma de adicionar as informações de mercado em um modelo mais consistente e com convergência dos preços físicos e futuros no momento em que o contrato futuro atinge sua maturidade.

\subsection{Razão de Hedge para Boi Gordo}

Diversos trabalhos propuseram-se a avaliar a razão ótima e efetividade do hedge no contrato futuro de boi gordo da B3 em diferentes períodos de tempo, para diferentes praças, e usando distintas abordagens metodológicas, o que leva a resultados divergentes.

Buscando analisar a cointegração e causalidade entre os preços de diferentes praças produtoras de boigordo no Sudeste e Centro-Oeste do Brasil, Zilli et al. (2008) testaram estas relações para preços físicos de três praças e os preços futuros de boi gordo na B3, mostrando que os preços futuros são causadores dos preços físicos nas referidas praças, indicando a importância dos agentes em considerarem operar contratos na bolsa de forma a reduzir o risco de variância nos retornos.

Oliveira Neto et al. (2009) analisaram as operações de hedge do boi gordo na B3 entre 1997 a 2007, considerando apenas o estado de Goiás. Usando o modelo tradicional de Myers e Thompson (1989) para o cálculo da razão ótima de hedge, os autores adicionaram uma variável dummy para identificar diferença no coeficiente estimado considerando os meses de safra e entressafra do abate neste estado. Seus resultados indicam uma razão de hedge elevada para esta região em ambos os períodos, sugerindo a intensa utilização do contrato, independente de seus períodos de vencimento.

Também utilizando o modelo de Myers e Thompson (1989), Freitas e Alves (2013) calculam o risco de base, a 
razão de hedge ótima e a efetividade do hedge para o contrato do boi gordo e o cross-hedge para bezerro usando o mesmo contrato, para quatro praças do estado de São Paulo, entre 2001 a 2010. Os autores também utilizaram uma variável dummy para diferenciar os períodos de safra e entressafra nestas praças, encontrando uma razão ótima de hedge par ao boi gordo na ordem de $45 \%$ em ambos os períodos, bem como uma efetividade superior a $70 \%$, sugerindo o uso do contrato por parte dos produtores paulistas. No entanto, ressalta-se que este trabalho analisou as praças que compõem o indicador de preços de referência no desenho do contrato futuro da B3, o que pode explicar a alta efetividade encontrada.

Resultados conflitantes aos apresentados foram encontrados por Monteiro et al. (2013), que diferente dos demais trabalhos, não utilizaram o modelo estático, de se calcular uma única razão ótima de hedge ao longo do tempo, mas de se propor uma razão dinâmica no tempo. Os autores utilizaram uma estimativa multivariada GARCH BEKK, considerando um termo de correção de erro, aplicando para seis praças, entre SP, MS, MT, PR e GO entre o período de 2002 a 2008. Os resultados indicam uma maior instabilidade nas operações de futuros (maior risco) se comparados à posição à vista. As razões de hedge, nos melhores dias, sugeriam operar no máximo $50 \%$ da produção, indicando, muitas vezes, valores negativos, deduzindo não efetividade para o hedge.

Amorim Neto (2015) analisou ambos modelos estáticos quanto dinâmicos, para determinar a razão ótima do hedge de boi gordo nas praças de Araçatuba e Goiânia, considerando o período de 2002 a 2013. O autor pôde concluir que a redução da variância dos retornos é maior quando se analisa os modelos estáticos, enquanto, os modelos dinâmicos mostram que a efetividade é menor.
Tal como Amorim Neto (2015) e complementando os estudos de Oliveira Neto et al. (2009), Monteiro et al. (2013) e Freitas e Alves (2013), o presente trabalho se propõe a aplicar modelos estáticos, baseado na metodologia de Myers e Thompson (1989), incluindo o mecanismo de correção de erro na existência de relação de longo prazo entre as variáveis de preços físicos e futuros defasadas e não defasadas, de forma a obter a razão ótima de hedge para o período mais recente (2012 a 2016), bem como propor o modelo dinâmico a partir dos resíduos dos modelos autorregressivos anteriormente estimados, utilizando-se da metodologia GARCH-BEKK, como explicitado na próxima seção do artigo, de forma a se averiguar a razão ótima de hedge no tempo e compreender sua amplitude em cada praça analisada. Além disso, o presente trabalho complementa os demais em razão de considerar sete praças produtoras nas principais áreas para a formação de preço do boi gordo no país.

Neste sentido, embasado em alguns dos estudos anteriormente apresentados, a presente pesquisa se propõe a testar algumas hipóteses, tal como representado no Quadro 1:

\section{ASPECTOS METODOLÓGICOS}

\subsection{Estimativa para o Hedge Estático}

O primeiro procedimento a ser testado para verificar a ordem de integração das séries consideradas foi através da metodologia de Dickey-Fuller Aumentado (DFA) que, conforme descrito por Enders (2004), permite identificar a presença ou não de raiz unitária. Concomitante, os critérios de Akaike (AIC) e Schwarz (BIC)² são considerados para a especificação da estrutura de defasagens de cada série.

QUADRO 1 - Hipóteses testadas a partir de resultados da literatura

\begin{tabular}{|c|c|c|}
\hline $1^{\mathrm{a}}$ Hipótese & $\begin{array}{l}\text { Tanto a razão de hedge de mínima variância, quanto à efetividade } \\
\text { do hedge no mercado futuro de boi gordo brasileiro é baixa. Isso } \\
\text { demonstra que operações no mercado de derivativos local tendem a } \\
\text { não reduzir os riscos de variação de preços da commodity. }\end{array}$ & $\begin{array}{l}\text { Adaptada a partir das conclusões } \\
\text { de Monteiro et. al. (2013) e } \\
\text { Mattos e Garcia (2006). }\end{array}$ \\
\hline 2a Hipótese & $\begin{array}{c}\text { Mesmo se tratando de commodity com sazonalidade, não será } \\
\text { possível verificar variações temporais significativas nas estimativas da } \\
\text { efetividade e razão de hedge dinâmico. }\end{array}$ & $\begin{array}{l}\text { Adaptada a partir das conclusões } \\
\text { de Monteiro et. al. (2013), } \\
\text { Amorim Neto (2015) e Oliveira } \\
\text { Neto et. al. (2009) }\end{array}$ \\
\hline
\end{tabular}

Fonte: Elaborado pelos autores

${ }^{2} \mathrm{O}$ critério AIC é uma medida relativa à qualidade de ajuste de um modelo estatístico paramétrico, estimado pelo método da máxima verossimilhança. Já o BIC é um critério de avaliação de modelos definido em termos da probabilidade a posteriori, tendo sua argumentação provada por método bayesiano. Apesar de conceitualmente diferentes acerca dos modelos de avaliação, utilizam o mesmo critério estatístico (máximo da função verossimilhança) como medida do ajustamento. Cabe ressaltar, porém, que possuem valores críticos diferentes (EMILIANO, 2009).

Organizações Rurais \& Agroindustriais, Lavras, v. 21, n. 1-3, p. 65-78, 2019 
O procedimento sequencial proposto por Enders (2004, p.203) a partir do teste DFA foi adotado com a finalidade de auxiliar na definição correta do modelo a ser utilizado no teste - com a presença ou não da constante e da tendência determinística. Assim, a equação 6 apresenta a formulação utilizada:

$\Delta y_{t}=\alpha+\beta t+\eta y_{t-1}+\sum_{i=1}^{p-1} \varphi_{i} \Delta y_{t-1}+e_{t}$

Uma vez identificadas as séries com raiz unitária e sua ordem de integração, o passo seguinte é o teste para a existência de cointegração entre as variáveis integradas, ou seja, a verificação de relações de longo prazo entre elas. A metodologia utilizada foi a proposta por Johansen (1988), que busca determinar o ranking (número de vetores de cointegração) através de um VAR de ordem $p$. Testase, portanto, a existência de $n$ vetores de cointegração, sendo indicada para modelos com mais de duas variáveis explicativas com raiz unitária.

O teste de Johansen é formulado para variáveis integradas de ordem 1, ou I(1), sendo sua formulação matemática expressa pela Equação (7) abaixo:

$\Delta y_{t}=\sum_{i=1}^{p-1} \Gamma_{i} \Delta y_{t-i}+\Pi y_{t-1}+\mu+\phi d_{t}+\varepsilon_{t}$

Em que $\mathbf{y}_{\mathbf{t}}$ é um vetor $(k \times 1)$ de variáveis $\mathrm{I}(1)$, e o $\varepsilon_{t} \sim N(0, \Sigma)$ e $E\left(\varepsilon t \varepsilon s^{\prime}\right)=0$ para qualquer $t$ diferente de $s$, sendo $\mathbf{d}_{\mathbf{t}}$ um vetor de variáveis binárias para captar a variação estacional. A matriz de coeficiente de $\mathbf{y}_{\mathbf{t}-1}$ (matriz П) possui as informações de longo prazo entre as variáveis. Assumindo que o posto desta matriz seja $r, \mathbf{y}_{\mathbf{t}}$ será estacionário se $r=k$. Caso $r=0$, П é uma matriz nula e a expressão será estacionária. Por fim, se $0<r<k$, existem matrizes $\alpha$ e $\beta$ de dimensão $k x r$, tais que $\boldsymbol{\Pi}=\boldsymbol{\alpha} \boldsymbol{\beta}$ ' e o vetor $\boldsymbol{\beta}$ ' $\boldsymbol{y}_{\boldsymbol{t}}$ é estacionário, existindo, assim, $\boldsymbol{r}$ vetores de cointegração, que são exatamente suas $r$ colunas (HARRIS, 1995).

Assumindo que os preços à vista e futuros são cointegrados, é esperado que estes possuam um equilíbrio de longo-prazo, após desvios de curto-prazo. As variáveis cointegradas podem ser representadas por um modelo de correção de erro, onde o erro corresponda às respostas aos desequilíbrios, tal como as expressões 8 e 9 a seguir (CHEN; LEE; SHRESTA, 2003; MATTOS; GARCIA, 2006).

$\Delta F_{t}=\alpha+\Pi z_{t-1}+\sum_{i=1}^{q} \beta_{i} \Delta F_{t-i}+\sum_{j=1}^{q} \gamma_{j} \Delta C_{t-j}+\varepsilon_{t}$
$\Delta C_{t}=\alpha+\Pi z_{t-1}+\sum_{i=1}^{q} \beta_{i} \Delta F_{t-i}+\sum_{j=1}^{q} \gamma_{j} \Delta C_{t-j}+\varepsilon_{t}$

em que $\Delta F_{t}$ e $\Delta C_{t}$ referem-se às primeiras diferenças dos preços futuros e à vista, respectivamente; $z_{t-1}$ reflete $\mathrm{o}$ desvio decorrente da defasagem do equilíbrio obtido pelo vetor de correção de erro П.

Segundo Mattos e Garcia (2006), se ambas as equações apresentarem o mesmo número de regressores de defasagem ${ }^{3}$, a estimação pelo método de mínimos quadrados ordinários pode ser realizada eficientemente. Ainda, na presença de cointegração, a razão de hedge é obtida pela estimação da equação 10 :

$\Delta C_{t}=\alpha+\Pi z_{t-1}+\varphi \Delta F_{t}+\sum_{i=1}^{q} \beta_{i} \Delta F_{t-i}+\sum_{i=1}^{q} \gamma_{j} \Delta C_{t-j}+\varepsilon_{t}$

em que $\phi$ representa a razão de hedge.

O passo seguinte se dá pelo cálculo da efetividade deste hedge $\left(E_{t}\right)$, a partir da comparação do risco entre a posição não-hedgeada com o risco da posição hedgeada, tal como expressão 11 .

$E_{t}=\frac{\operatorname{Var}\left(\Delta C_{t}^{u}\right)-\operatorname{Var}\left(\Delta C_{t}^{h}\right)}{\operatorname{Var}\left(\Delta C_{t}^{u}\right)}=1-\frac{\operatorname{Var}\left(\Delta C_{t}^{h}\right)}{\operatorname{Var}\left(\Delta C_{t}^{u}\right)}$

em que $\operatorname{Var}\left(\Delta C_{t}^{h}\right)$ corresponde à variância condicional da mudança no preço à vista com adoção de hedge na proporção ótima; e $\operatorname{Var}\left(\Delta C_{t}^{h}\right)$ corresponde à variância condicional da mudança no preço à vista sem a realização do hedge; $E_{t}$ mensura a redução percentual na variância condicional na mudança do preço à vista quando ocorre o hedge. Assim, na presença de hedge, a variância condicional desta mudança pode ser estimada conforme equação 12 .

$\operatorname{Var}\left(\Delta C_{t}^{h}\right)=M S E=\frac{S S E}{T-K}$

sendo MSE o quadrado médio residual (mean squared error) e SSE a soma dos quadrados dos resíduos (sum of squared errors) gerados pela estimação da expressão (10); $T$ corresponde ao número de observações; e $K$ corresponde ao número de variáveis da equação mais a constante.

De outra forma, tal como expressam Mattos e Garcia (2006), na ausência do hedge, a variância

${ }^{3}$ Testado pelos critérios de $\mathrm{AIC}$ e SBC, para uma versão multiequacional. 
condicional da mudança no preço à vista é obtida pelo erro quadrado médio, a partir da equação 13 .

$\Delta C_{t}=\alpha^{\prime}+\Pi^{\prime} z_{t-1}+\sum_{i=1}^{q} \beta_{i}^{\prime} \Delta F_{t-i}+\sum_{j=1}^{q} \gamma^{\prime}{ }_{j} \Delta C_{t-j}+\varepsilon^{\prime}{ }_{t}$

Note que, neste caso, o termo correspondente à razão de hedge na equação (10) é excluído em virtude da ausência de operação de hedge.

\subsection{Estimativa para o Hedge Dinâmico}

O hedge dinâmico considera os efeitos das variações temporais entre os preços spot e futuros do boi gordo no Brasil. Ou seja, ao invés de estimar uma efetividade e razão de hedge durante todo o período, estimam-se as possíveis variações da efetividade e razão de hedge. Assim é possível analisar os momentos em que se deve ter uma maior proteção no mercado futuro. Para esta estimativa foi considerado o modelo multivariado Diagonal Bekk, proposto por Engle e Kroner (1995), como estimador da covariância condicional, considerando as variáveis do modelo de correção de erro.

O modelo Diagonal Bekk é uma extensão do modelo diagonal Vech e Bekk propostos por Bollerlev et. al. (1988) e Baba et. al. (1991). Nesta formulação, busca-se modelar a matriz de covariância $\boldsymbol{H}_{\boldsymbol{t}}$ condicionada no tempo t, sendo definida conforme expressão 14 .

$H_{t}=\Omega \Omega^{\prime}+\mathrm{A} \epsilon_{t-1} \epsilon_{t-1}^{\prime} A^{\prime}+B H_{t-1} B^{\prime}$

em que $H_{t}$ é a matriz de covariância, tendo as covariâncias expressa nas diagonais e o restante com as variâncias. $\boldsymbol{A}, \boldsymbol{B}$ e $\boldsymbol{\Omega}$ são matrizes simétricas $(N \times N)$ de parâmetros, estimados pelo método de máxima verossimilhança. Neste sentido, as estimativas da efetividade e razão de hedge em sua forma dinâmica seguem o mesmo formato da estática, como descrita na seção anterior, porém, incorporando as extensões do modelo Diagonal Bekk para o cômpito das variações temporais dos valores estimados.

\subsection{Dados da Pesquisa}

São considerados os preços físicos diários em sete praças que representam importantes regiões produtoras, as quais referentes à região de Cuiabá (MT), Campo Grande (MS), Dourados (MS), Goiânia (GO), Rio Verde (GO), Araçatuba (SP) e Noroeste do Paraná. Pretende-se, dessa forma, avaliar se há diferenças significativas para o hedger, a depender de sua região de referência, e que possam estimular (desestimular) tal operação. O período de análise constituise entre janeiro de 2012 a setembro de 2016, com dados diários, somando-se 1.156 observações para cada praça. Os dados de preços físicos utilizados têm como referência os preços regionais obtidos junto ao Cepea/Esalq, enquanto os preços futuros são referentes ao contrato de boi gordo da B3. Para as simulações, as séries de preços futuros foram deflacionadas pelo Índice de Preço ao Consumidor Amplo - IPCA, com base em setembro de 2016, e transformadas considerando o primeiro dia do mês de vencimento de cada contrato para rolagem da série de futuros ${ }^{4}$.

\section{RESULTADOS E DISCUSSÃO}

Primeiramente, como forma de permitir melhor interpretação dos dados utilizados, apresenta-se na Tabela 1, o resultado das estatísticas descritivas para as séries de preços físicos e futuros em nível, sendo possível notar valores próximos nos limites máximos e mínimos, na média e, consequentemente, na dispersão dos preços físicos e futuros.

Os valores dos testes de normalidade de JarqueBera evidenciam, para todas as praças e futuro, o que já era esperado em séries financeiras, a não normalidade dos dados. Isso ocorre porque as séries são assimétricas (Assimetria $\neq 0$ ) e curtoses diferentes de 3 . Nas séries em análise, a curtose é inferior a três, o que caracteriza uma distribuição platicúrtica. Já as séries em primeira possuem uma característica leptocúrtica. Mais evidente nos preços de ajuste dos contratos futuros (BGI). Essas definições estatísticas são apresentadas em Casella e Berger (2011).

Os resultados dos testes de raiz unitária apontaram que as séries em nível possuem raízes unitárias, uma vez que os coeficientes dos testes ADF aceitam a hipótese nula de raiz unitária na série. Já as séries em primeira diferença ocorreram o inverso. A hipótese nula de raiz unitária foi rejeita nos testes ADF (Tabela 2).

Em síntese, as séries em nível não são estacionárias, mas em primeira diferença, sim, devendo, assim, optar-se pelo segundo passo na análise autorregressiva, de acordo com Granger e Newbold (1974).

Como passo seguinte, foi testada a relação de longo prazo entre os preços físicos de cada praça e os preços futuros de boi gordo da B3. Para isso foi aplicado o teste de cointegração proposto por Johansen (1988). Os resultados deste teste estão expressos na Tabela 3.

\footnotetext{
${ }^{4}$ Em razão dos vencimentos mensais de cada contrato, estimações adicionais assumindo dados de preços futuros considerando o $2^{\circ}$ vencimento $(D+60)$ e $3^{\circ}$ vencimento $(\mathrm{D}+90)$ foram testadas, constatando-se pouca variação na efetividade e razão ótima do hedge.
} 
TABELA 1 - Estatística descritiva dos preços futuros e físicos de boi gordo, 2012-2016

\begin{tabular}{ccccccccc}
\hline Em nível & Futuro & Araçatuba & Campo Grande & Cuiabá & Dourados & Goiânia & Noroeste do Paraná & Rio Verde \\
\hline Média & 144,81 & 144,57 & 135,84 & 129,56 & 135,86 & 133,40 & 143,14 & 133,39 \\
Mediana & 145,17 & 144,63 & 139,45 & 133,01 & 139,58 & 135,55 & 142,32 & 135,61 \\
Máximo & 174,48 & 173,82 & 164,29 & 159,64 & 164,66 & 162,63 & 170,59 & 162,62 \\
Mínimo & 118,92 & 115,15 & 110,54 & 106,87 & 110,47 & 107,34 & 118,96 & 106,59 \\
Desvio Padrão & 16,43 & 16,32 & 15,23 & 14,67 & 15,20 & 15,77 & 15,59 & 15,66 \\
Assimetria & 0,04 & 0,03 & 0,16 & 0,24 & 0,15 & 0,09 & 0,13 & 0,12 \\
Curtose & 1,50 & 1,54 & 1,79 & 1,81 & 1,78 & 1,68 & 1,51 & 1,72 \\
Jarque-Bera & 108,24 & 102,58 & 75,80 & 79,14 & 76,67 & 85,50 & 109,63 & 81,70 \\
p-valor & 0,00 & 0,00 & 0,00 & 0,00 & 0,00 & 0,00 & 0,00 & 0,00 \\
no observações & 1156 & 1156 & 1156 & 1156 & 1156 & 1156 & 1156 & 1156 \\
\hline
\end{tabular}

Fonte: Dados da pesquisa

TABELA 2 - Resultados dos testes de raiz unitária de Dickey Fuller Aumentado (ADF)

\begin{tabular}{ccccccccc}
\hline Teste ADF & Futuro & Araçatuba & Campo Grande & Cuiabá & Dourados & Goiânia & Noroeste do Paraná & Rio Verde \\
\hline$\tau \tau$ & $-1,179$ & $-1,166$ & $-0,829$ & $-1,033$ & $-0,844$ & $-1,325$ & $-0,932$ & $-1,629$ \\
$\tau \mu$ & $-0,885$ & $-0,801$ & $-0,785$ & $-0,829$ & $-0,793$ & $-1,085$ & $-0,745$ & $-1,162$ \\
$\tau$ & 0,319 & 0,413 & 0,560 & 0,340 & 0,507 & 0,262 & 0,536 & 0,247 \\
$\Delta \tau$ & $-37,088^{*}$ & $-24,871^{*}$ & $-39,054^{*}$ & $-44,604^{*}$ & $-39,137^{*}$ & $-11,541^{*}$ & $-41,390^{*}$ & $-9,729 *$ \\
\hline
\end{tabular}

Fonte: Dados da pesquisa

Nota: $(*)$ indica a significância a 1\%. Os limites assintóticos dos testes de estacionariedade (raiz unitária) para o nível de significância de $1 \%$, considerando, respectivamente, os efeitos de constante e tendência determinística, constante e sem tendência determinística, sem constante e sem tendência determinística (em nível e na primeira diferença), são: -3,966, -3,436 e -2,567.

TABELA3 - Resultados do teste de cointegração de Johansen entre os preços do boi gordo futuro e as praças comercializadoras

\begin{tabular}{|c|c|c|c|c|c|c|}
\hline Praça & VEC Lag & Hipótese & Estat. Traço & Valor Crítico (5\%) & Estat. Max. & Valor Crítico (5\%) \\
\hline \multirow{2}{*}{ Araçatuba } & \multirow{2}{*}{3} & $H_{0}: r$ & 128,94 & 15,49 & 128,10 & 14,26 \\
\hline & & $H_{0}: r$ & 0,84 & 3,84 & 0,84 & 3,84 \\
\hline \multirow{2}{*}{ Campo Grande } & \multirow{2}{*}{1} & $H_{0}: r$ & 18,44 & 15,49 & 17,93 & 14,26 \\
\hline & & $H_{0}: r$ & 0,51 & 3,84 & 0,51 & 3,84 \\
\hline \multirow{2}{*}{ Cuiabá } & \multirow{2}{*}{1} & $H_{0}: r$ & 27,03 & 15,49 & 26,32 & 14,26 \\
\hline & & $H_{0}: r$ & 0,71 & 3,84 & 0,71 & 3,84 \\
\hline \multirow{2}{*}{ Dourados } & \multirow{2}{*}{1} & $H_{0}: r$ & 22,54 & 15,49 & 22,02 & 14,26 \\
\hline & & $H_{0}: r$ & 0,52 & 3,84 & 0,52 & 3,84 \\
\hline \multirow[b]{2}{*}{ Goiânia } & \multirow{2}{*}{3} & $H_{0}: r$ & 17,81 & 15,49 & 17,03 & 14,26 \\
\hline & & $H_{0}: r$ & 0,78 & 3,84 & 0,78 & 3,84 \\
\hline \multirow{2}{*}{ Noroeste do Paraná } & \multirow{2}{*}{1} & $H_{0}: r$ & 38,08 & 15,49 & 37,44 & 14,26 \\
\hline & & $H_{0}: r$ & 0,63 & 3,84 & 0,63 & 3,84 \\
\hline \multirow{2}{*}{ Rio Verde } & \multirow[b]{2}{*}{2} & $H_{0}: r$ & 23,48 & 15,49 & 22,84 & 14,26 \\
\hline & & $H_{0}: r$ & 0,64 & 3,84 & 0,64 & 3,84 \\
\hline
\end{tabular}

Fonte: Dados da pesquisa

Nota: O critério de escolha dos lags do VEC foi estabelecido conforme resultados dos AIC, BIC e HK, como discute Lutkepohl (1991). Os testes foram aplicados para modelo linear com intercepto e tendência determinística.

Organizações Rurais \& Agroindustriais, Lavras, v. 21, n. 1-3, p. 65-78, 2019 
Pode-se constatar, pelo teste de cointegração, que em todas as praças a hipótese nula de ausência de vetores de cointegração é rejeitada ao nível de 5\%, tanto na estatística traço, quanto na estatística máximo autovalor. No entanto, a hipótese nula de existência de vetores é aceita em todas os casos, sugerindo a existência de um vetor de cointegração. Isso evidencia que as séries de preços do boi gordo nas praças comercializadoras são cointegradas. De acordo com Engle e Granger (1987) isso confirma a necessidade de utilização de vetores de correção de erros para estimação das regressões.

A Tabela 4 apresenta os resultados dos modelos de correção de erro descrito na seção 3. De forma geral as regressões para estimativa das razões de hedge foram coerentes com os preceitos econométricos.

A estatística $\mathrm{F}$ foi significativa a $1 \%$ em todos os níveis, sugerindo que os ajustes de equilíbrio podem ser feitos tanto para as séries de preços à vista, como preços futuros. A estatística de Durbin Watson ficou entre os limites assintóticos, o que a coloca na área de aceitação da hipótese nula de independência dos resíduos. Assim, podese afirmar que não há o efeito de autocorrelação serial.

Em relação aos resultados estimados no modelo estático para a razão ótima de hedge e efetividade para o mercado de boi gordo no Brasil, nota-se que este contrato não tem sido eficiente para atender os hedgers de diferentes praças do país, como pode-se observar na Tabela 5.
Nota-se que os percentuais de hedge nas praças comercializadoras são inferiores a $25 \%$ da produção total, patamar pouco significativo para um setor de alta expressão no agronegócio e com produção alastrada por todo o país. De todas as praças analisadas, Araçatuba foi a que obteve o maior índice de razão, provavelmente em razão desta praça compor o indicador de preços CEPEA/Esalq, usado como referência no contrato futuro. Os valores, de forma geral, estão coerentes aos estudos de Mattos e Garcia (2006) que teve a razão de hedge para os contratos de boi gordo em torno de 7\%, e Monteiro et. al. (2013), com valores inferiores a 50\% para as praças analisadas. No entanto, foi diferente do estudo de Amorim Neto (2015) que ficou em torno de $45 \%$ nas praças de Araçatuba e Goiânia, e Oliveira Neto et. al. (2009) que ficaram acima de $95 \%$ para as praças no estado de Goiás.

As evidências aqui confirmam a hipótese de não efetividade do hedge no Brasil, conforme levantamento de Monteiro et. al. (2013) e Mattos e Garcia (2006) e apresentadas anteriormente no Quadro 1. Os resultados da Tabela 5 mostram que em nenhuma praça a efetividade do hedge superou 5\%, mesmo em Araçatuba, que apresentou a maior razão de hedge. A baixa efetividade do hedge pode estar relacionada ao percentual de especulação inerente aos contratos. Quanto maior o número de especuladores no mercado, menor será a relação entre os preços físicos e futuro. A redução do risco de mercado por meio de contratos

TABELA 4 - Representação do termo de correção de erro dos preços cointegrados à vista e futuros de boi gordo

\begin{tabular}{cccccccc}
\hline & Araçatuba & Campo Grande & Cuiabá & Dourados & Goiânia & Noroeste Paraná & Rio Verde \\
\hline \multirow{2}{*}{ Constante } & 0,01 & 0,01 & 0,01 & 0,01 & 0,01 & 0,01 & 0,01 \\
& $(0,28)$ & $(0,60)$ & $(0,39)$ & $(0,56)$ & $(0,27)$ & $(0,60)$ & $(0,35)$ \\
VEC & $-0,27^{*}$ & $-0,02^{*}$ & $-0,03^{*}$ & $-0,03^{*}$ & $-0,04^{*}$ & $-0,04^{*}$ & $-0,03^{*}$ \\
& $(-13,99)$ & $(-3,51)$ & $(-5,08)$ & $(-4,18)$ & $(-5,07)$ & $(-6,00)$ & $(-4,70)$ \\
$\beta_{1}$ & $0,25^{*}$ & $0,16^{*}$ & $0,11^{*}$ & $0,13^{*}$ & $0,19^{*}$ & $0,10^{*}$ & $0,14^{*}$ \\
& $(6,77)$ & $(6,72)$ & $(3,79)$ & $(5,28)$ & $(7,19)$ & $(4,00)$ & $(6,16)$ \\
$\gamma_{1}$ & $-0,03$ & $-0,15^{*}$ & $-0,26^{*}$ & $-0,14^{*}$ & $0,06^{* *}$ & $-0,19^{*}$ & 0,05 \\
& $(-1,28)$ & $(-5,19)$ & $(-9,34)$ & $(-4,85)$ & $(2,04)$ & $(-6,82)$ & $(1,79)$ \\
$\gamma_{2}$ & $-0,04$ & 0,00 & 0,01 & $-0,01$ & $0,08^{*}$ & $-0,01$ & $0,12^{*}$ \\
\hline $\mathrm{R}^{2}$ & $(-1,09)$ & $(-0,10)$ & $(0,40)$ & $(-0,53)$ & $(3,06)$ & $(-0,46)$ & $(5,12)$ \\
$\mathrm{R}^{2}$ Ajust. & 0,16 & 0,06 & 0,10 & 0,06 & 0,08 & 0,08 & 0,08 \\
Estat. F & 0,16 & 0,06 & 0,10 & 0,05 & 0,07 & 0,08 & 0,07 \\
p-valor & 55,83 & 19,62 & 32,78 & 16,86 & 23,28 & 24,46 & 24,07 \\
Estat. DW & 0,00 & 0,00 & 0,00 & 0,00 & 0,00 & 0,00 & 0,00 \\
\hline
\end{tabular}

Fonte: Dados da pesquisa

Nota: A estatística t está entre parênteses. (*) significativo a 1\% e (**) significativo a 5\%. Os limites assintóticos são de 2,576 e 1,960 para os níveis de $1 \%$ e $5 \%$ respectivamente. As defasagens dos coeficientes gama correspondem às apresentadas na Tabela 3 . Os limites assintóticos para o teste DW são entre 1,59 (du) e 2,84 (4-dl) para o nível de 1\%. 
futuros só é permitida quando se tem altos índices de efetividade. Neste caso, percebe-se que o mercado futuro de boi gordo na B3 não tem sido eficiente para redução dos riscos por parte dos hedgers, sobretudo produtores.

$\mathrm{Na}$ Tabela 6, apresentam-se os resultados das estimativas das covariâncias e correlações temporais por meio da regressão Diagonal Bekk.

De forma geral os ajustamentos para as covariâncias e variâncias dos resíduos do modelo de correção de erros foi significativa para os modelos Diagonal Bekk, uma vez que os parâmetros foram significativos a $1 \%$ em sua maioria.

Os coeficientes significativos dos parâmetros das diagonais $A$ indicam que os erros passados () explicam as covariâncias presentes. Em outras palavras, a volatilidade condicional dos preços físicos depende de choques passados ou inovações dos erros. Ou seja, eles medem a dependência da volatilidade dos preços físicos com as inovações passadas. Esse coeficiente é também denominado como ARCH.

TABELA 5 - Resultados das estimações da razão de hedge ótimas e efetividade do hedge nas operações com futuros de boi gordo por praça produtora

\begin{tabular}{cccccc}
\hline Praça & Razão Ótima & Estat. F & Valor p & Estat. DW & Efetividade \\
\hline Araçatuba & $24,7 \%$ & 55,83 & 0,00 & 2,28 & $3,8 \%$ \\
Campo Grande & $16,0 \%$ & 19,62 & 0,00 & 1,99 & $3,8 \%$ \\
Cuiabá & $10,9 \%$ & 32,78 & 0,00 & 2,03 & $1,2 \%$ \\
Dourados & $12,8 \%$ & 16,86 & 0,00 & 2,01 & $2,4 \%$ \\
Goiânia & $18,7 \%$ & 23,28 & 0,00 & 2,46 & $4,3 \%$ \\
Noroeste do Paraná & $9,9 \%$ & 24,46 & 0,00 & 2,01 & $1,4 \%$ \\
Rio Verde & $14,1 \%$ & 24,07 & 0,00 & 2,31 & $3,2 \%$ \\
\hline
\end{tabular}

Fonte: Dados da pesquisa

TABELA 6 - Estimativas do modelo Diagonal Bekk $(1,1)$

\begin{tabular}{cccccccc}
\hline & Araçatuba & Campo Grande & Cuiabá & Dourados & Goiânia & Noroeste do Paraná & Rio Verde \\
\hline \multirow{2}{*}{$\Omega(1,1)$} & $0,04^{*}$ & $0,64^{* *}$ & $0,07^{*}$ & $0,73^{*}$ & $0,72^{*}$ & $0,71^{*}$ & $0,66^{*}$ \\
& $(2,71)$ & $(2,06)$ & $(3,27)$ & $(3,42)$ & $(30,06)$ & $(8,50)$ & $(3,19)$ \\
$\Omega(1,2)$ & $0,01^{*}$ & 0,08 & 0,02 & 0,12 & 0,14 & 0,06 & 0,07 \\
& $(4,86)$ & $(1,31)$ & $(0,40)$ & $(1,02)$ & $(1,48)$ & $(1,42)$ & $(1,50)$ \\
$\Omega(2,2)$ & $0,02^{*}$ & $0,10^{*}$ & 0,22 & $0,04^{*}$ & $0,08^{*}$ & $0,06^{*}$ & $0,03^{*}$ \\
& $(3,35)$ & $(4,85)$ & $(0,25)$ & $(3,97)$ & $(4,16)$ & $(5,03)$ & $(5,50)$ \\
$A(1,1)$ & $0,04^{* *}$ & $0,13^{*}$ & $-0,31^{*}$ & $-0,10^{*}$ & $0,18^{*}$ & $-0,20^{*}$ & $0,17^{*}$ \\
& $(2,36)$ & $(6,68)$ & $(-7,42)$ & $(-2,60)$ & $(4,56)$ & $(-4,10)$ & $(3,88)$ \\
$A(2,2)$ & $0,34^{*}$ & $0,34^{*}$ & 0,01 & $0,33^{*}$ & $0,27^{*}$ & $0,39^{*}$ & $0,27^{*}$ \\
& $(16,19)$ & $(11,74)$ & $(0,21)$ & $(14,50)$ & $(8,59)$ & $(12,86)$ & $(10,79)$ \\
$B(1,1)$ & $0,97^{*}$ & 0,33 & $0,90^{*}$ & $-0,12$ & $-0,02$ & 0,08 & 0,28 \\
& $(93,92)$ & $(0,51)$ & $(34,58)$ & $(-0,10)$ & $(-0,03)$ & $(0,11)$ & $(0,57)$ \\
$B(2,2)$ & $0,93^{*}$ & $0,82^{*}$ & 0,84 & $0,91 *$ & $0,90^{*}$ & $0,87^{*}$ & $0,93 *$ \\
Log Vero. & $(100,88)$ & $(24,64)$ & $(1,22)$ & $(64,46)$ & $(43,50)$ & $(43,97)$ & $(88,40)$ \\
Schwarz & $-3022,62$ & $-2633,00$ & $-2897,11$ & $-2656,37$ & $-2771,10$ & $-2684,73$ & $-2607,01$ \\
& 5,3394 & 4,6549 & 5,1126 & 4,6954 & 4,9027 & 4,7446 & 4,6138 \\
\hline
\end{tabular}

Fonte: Dados da pesquisa

Nota: A estatística t está entre parênteses. (*) significativo a $1 \%$ e (**) significativo a 5\%. Os limites assintóticos são de 2,576 e 1,960 para os níveis de $1 \%$ e $5 \%$ respectivamente. 
Em relação ao coeficiente $B$, se significativo, e covariâncias passadas () explicam as covariâncias presentes $\left(H_{t}\right)$ das séries. Isso evidencia que as volatilidades dos preços físicos são explicadas pelas volatilidades passadas dos preços futuros. Os valores das diagonais $A(i, n)$ e $B(i, n)$ indicam os chamados autotransbordamentos de volatilidade (own-volatility spillover). Ou seja, como os valores passados das volatilidades de um mercado podem explicar as volatilidades de outros.

A Tabela 7 apresenta as estatísticas descritivas das séries de efetividade e razão ótima considerando o hedge dinâmico no tempo das praças comercializadoras. Já a Figura 1 exibe os gráficos das séries tanto para o hedge estativo quanto dinâmico.

Assim como no hedge estático, no hedge dinâmico a efetividade também foi baixa, estando, em quase todas as praças, inferior a 5\%. No entanto, como a ideia é analisar as possíveis alterações do hedge no tempo, os valores máximos também estão abaixo de uma perspectiva de redução de risco por meio de contratos de derivativos. Em todas as praças, o período de maior efetividade não superou $50 \%$ de redução de risco. As praças de Araçatuba e Campo Grande foram as que obtiveram os maiores resultados, mas ambos abaixo de 50\%.

A média da razão de hedge ficou bem próxima da estimativa por hedge estático, valores inferiores a $25 \%$. Os valores máximos, mesmo na praça de Araçatuba, que compõe o indicador Cepea/Esalq, também foram pequenos. Não foi possível constatar nenhuma praça em que, para a redução do risco, fosse aplicada uma trava superior a $70 \%$ da produção. Nota-se, também, que as praças com maior razão de hedge no modelo estático apresentaram maiores desvios na razão de hedge dinâmica, como Araçatuba, enquanto praças com menores razões de hedge estática, como Cuiabá, a variação na razão de hedge dinâmica apresentaramse baixa.

Na Figura 1 é possível observar a relação dos resultados obtidos a partir das análises do hedge estático e do hedge dinâmico. Observa-se que pela análise dinâmica, as dispersões dos pontos podem, em muitos casos, se distanciar significativamente dos pontos estáticos estimados. A única exceção observada é para a praça de Cuiabá, em que a dispersão dos pontos estimados pelo modelo dinâmico é baixa em comparação com a estimada na análise estática. Nas demais praças, por exemplo, essa diferença é mais visível uma vez que, enquanto a análise estática demonstra efetividade positiva, embora baixa, a análise dinâmica ilustra que em muitos momentos não existe efetividade alguma na operação com contrato futuro de boi gordo. Em outros momentos, porém a efetividade pode ultrapassar valores acima dos $40 \%$ em algumas praças (pelo hedge dinâmico), como Araçatuba, Campo Grande e Rio Verde, os quais bem superiores aos valores obtidos no modelo estático. A lógica inversa se observa ao avaliar os pontos inferiores calculados pelo modelo dinâmico.

TABELA 7 - Estatística descritiva das séries de razão ótima e efetividade do hedge dinâmicos

\begin{tabular}{cccccccc}
\hline Razão Ótima & Araçatuba & Campo Grande & Cuiabá & Dourado & Goiânia & Noroeste do Paraná & Rio Verde \\
\hline Média & $24,23 \%$ & $15,40 \%$ & $11,11 \%$ & $13,95 \%$ & $18,96 \%$ & $7,70 \%$ & $14,37 \%$ \\
Mediana & $23,45 \%$ & $14,88 \%$ & $11,32 \%$ & $14,37 \%$ & $18,71 \%$ & $8,52 \%$ & $13,96 \%$ \\
Máximo & $66,90 \%$ & $55,12 \%$ & $13,65 \%$ & $36,32 \%$ & $51,10 \%$ & $59,55 \%$ & $45,53 \%$ \\
Mínimo & $11,04 \%$ & $-12,98 \%$ & $-16,00 \%$ & $-13,56 \%$ & $-2,35 \%$ & $-46,65 \%$ & $0,46 \%$ \\
Desvio Padrão & $4,92 \%$ & $4,01 \%$ & $1,40 \%$ & $2,82 \%$ & $3,71 \%$ & $6,00 \%$ & $3,31 \%$ \\
Efetividade & & & & & & $2,05 \%$ & $4,03 \%$ \\
Média & $5,10 \%$ & $3,50 \%$ & $1,49 \%$ & $3,17 \%$ & $4,64 \%$ & $2,09 \%$ & $3,53 \%$ \\
Mediana & $6,33 \%$ & $3,95 \%$ & $1,53 \%$ & $3,30 \%$ & $5,14 \%$ & $20,17 \%$ & $26,59 \%$ \\
Máximo & $47,56 \%$ & $47,42 \%$ & $12,87 \%$ & $22,27 \%$ & $32,59 \%$ & $-24,50 \%$ & $-10,99 \%$ \\
Mínimo & $-33,09 \%$ & $-55,69 \%$ & $-16,85 \%$ & $-20,40 \%$ & $-15,71 \%$ & $6,46 \%$ & $5,30 \%$ \\
Desvio Padrão & $9,60 \%$ & $6,70 \%$ & $3,57 \%$ & $5,09 \%$ & $6,05 \%$ & & 6 \\
\hline
\end{tabular}

Fonte: Dados da pesquisa 


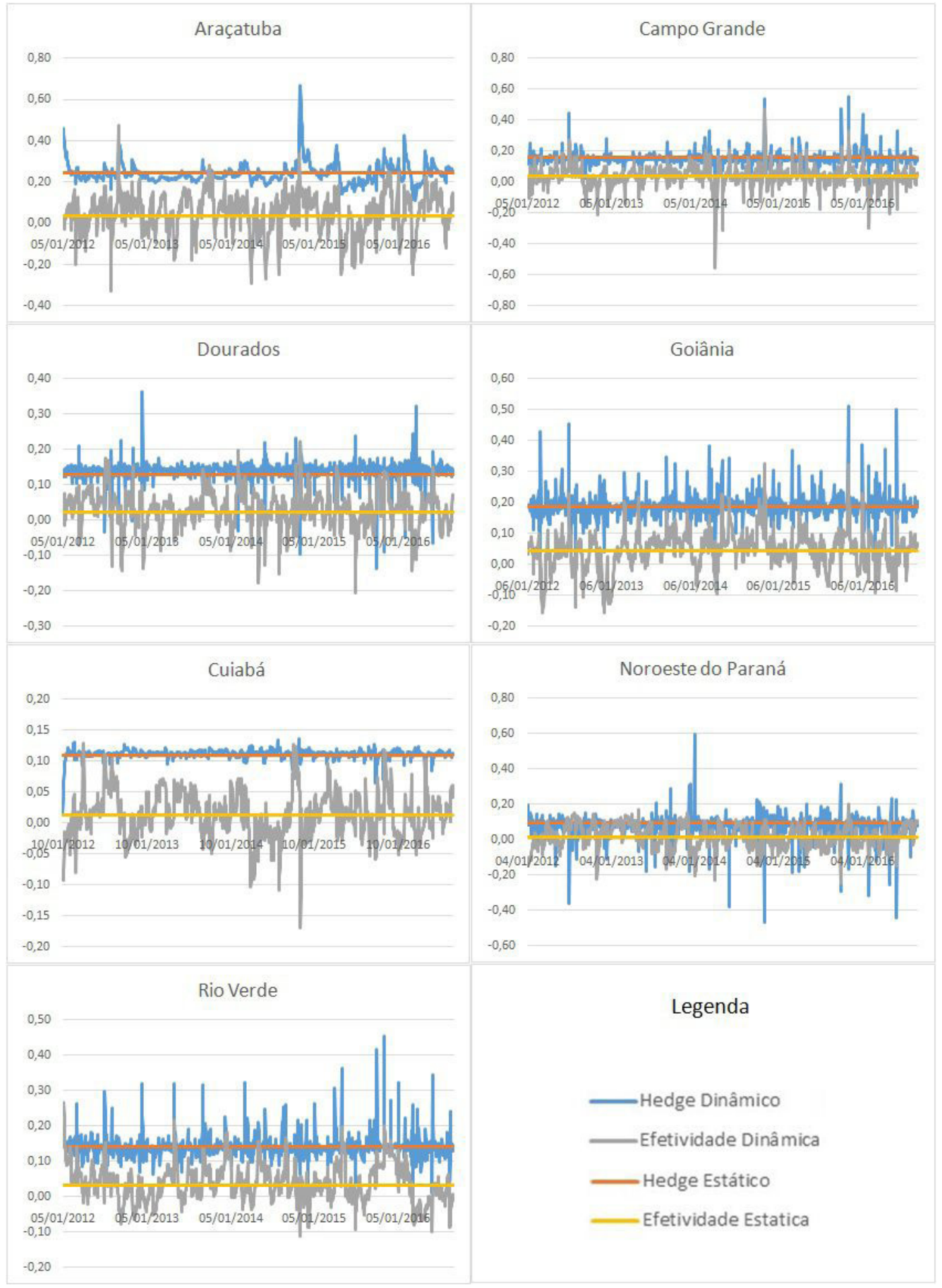

FIGURA 1 - Comportamento da razão ótima e efetividade do hedge estáticos e dinâmicos por praça produtora, 2012-2016

Fonte: Dados da pesquisa

Organizações Rurais \& Agroindustriais, Lavras, v. 21, n. 1-3, p. 65-78, 2019 


\section{CONSIDERAÇÕES FINAIS}

A presente pesquisa teve por objetivo analisar o desempenho do hedge no mercado futuro de boi gordo no Brasil, considerando suas principais praças produtoras. Para isso, foi avaliado a possibilidade de realização de um hedge estático, sem considerar as alterações temporais, e o hedge dinâmico, estimando-se as efetividades do hedge e sua razão de mínima variância.

De acordo com as hipóteses teóricas previamente estabelecidas e embasadas em outros estudos, observouse que ambas foram aceitas. Isso indica que a realidade do mercado de boi gordo nacional, no que diz respeito à efetividade de seu contrato futuro doméstico, não se alterou nas últimas décadas.

Em relação à primeira hipótese, de não efetividade e baixa eficiência do hedge do boi gordo, os resultados apontaram que os contratos futuros de boi gordo na B3 não geram efetividade e proteção para redução da volatilidade dos preços nos mercados físicos em análise. Isso geralmente ocorre quando o mercado possui um volume elevado de especuladores, o que possibilita maior volatilidade e descolamento os preços futuros do spot, ou seja, a dinâmica dos preços futuros tem respondido mais a um comportamento especulativo, enquanto os mercados físicos apresentam variações concernentes às variações na oferta e demanda da própria commodity. Complementarmente, na análise posterior do hedge dinâmico, também se observou a não efetividade ao longo de todo o período temporal considerado. Mesmo na praça de Araçatuba, uma das regiões formadoras do indicador Cepea/Esalq, a razão de mínima variância foi pequena. Isso corrobora a ideia de existência de um efeito especulativo forte no mercado futuro de boi gordo da B3.

Em relação à segunda hipótese teórica, sobre a variação temporal das estimativas de efetividade e razão, considerando o hedge dinâmico, o resultado do estudo também pode aceitá-la, de acordo com os trabalhos de Monteiro et. al. (2013), Amorim Neto (2015) e Oliveira Neto et. al. (2009). Nota-se que o comportamento dos desvios na razão de hedge dinâmica não apresentou um comportamento cíclico, bem como seus pontos de máximo e mínimo. Ainda, ressalta-se que os desvios foram diferentes, sendo maiores nas praças com maior razão de hedge de mínima variância.

Desta maneira, considerando os resultados apresentados, entende-se que esta pesquisa fornece subsídios para uma maior discussão acerca do papel dos contratos futuros de boi gordo no Brasil como ferramenta efetiva de redução de risco. Sua contribuição serve para que os pecuaristas avaliem, de antemão, se a estratégia de hedge realmente será efetiva e cumprirá o propósito de redução de risco.

Ademais, de forma a complementar as evidências encontradas neste estudo, sugere-se que pesquisas futuras possam realizar uma análise do comportamento da efetividade e razão de hedge durante os anos, buscando avaliar possíveis tendências e questões cíclicas ou sazonais. Outro problema de pesquisa pertinente seria analisar o aspecto dos operadores destes contratos. Mesmo com a B3 divulgando o percentual de hedgers, as evidências de que haja um volume grande de especuladores é grande. Dessa forma, através de uma pesquisa por método survey seria possível identificar com mais exatidão quem são os participantes.

\section{REFERÊNCIAS}

AMORIM NETO, C. S. Efetividade do hedge para o boi gordo com contratos futuros da BM\&FBOVESPA: análise para o estado de São Paulo e Goiás. 2015. 49 f. Dissertação (Mestrado em Economia Aplicada) - Escola Superior de Agricultura "Luiz de Queiroz" da Universidade de São Paulo, Piracicaba, 2015.

BABA, Y.; ENGLE, R. F.; KRAFT, D. F.; KRONER, K. F. Multivariate Simultaneous Generalized ARCH. San Diego: UCSD, Dept. of Economics, 1991.

BOLLERSLEV, T. Modelling the coherence in short-run nominal exchange rates: A multivariate generalized ARCH model. Review of Economics and Statistics, Boston, v. 72, p. 498-505, 1990.

BOLLERSLEV, T.; ENGLE, R. F.; WOOLDRIDGE, J. M. A capital asset pricing model with time-varying covariances. The Journal of Political Economy, Chicago, v. 96, p. 116-131, 1988.

CASELLA, G.; BERGER, R. L. Inferência estatística. 2. ed. São Paulo: Cengage Learning, 2011.

CHEN, S.; LEE, C.; SHRESTHA, K. Futures hedge ratios: a review. The Quarterly Review of Economics and Finance, Urbana-Champaign, v. 43, n. 1, p. 433465, 2003.

Organizações Rurais \& Agroindustriais, Lavras, v. 21, n. 1-3, p. 65-78, 2019 
CRUZ JÚNIOR, J. C. Modelo de razão de hedge ótima e percepção subjetiva de risco nos mercados futuros. 2009. 100 f. Tese (Doutorado em Economia Aplicada) - Escola Superior de Agricultura "Luiz de Queiroz", Universidade de São Paulo, Piracicaba, 2009.

DICKEY, D. A.; FULLER, W. A. Likelihood ratio statistics for autoregressive time series with a unit root. Econometrica, Chicago, v. 49, n. 4, p. 1057-1072, jul. 1981.

EDERINGTON, L. H. The hedging performance of the new futures markets. The Journal of Finance, Pittsburgh, v. 34, n. 1, p. 157-170, mar. 1979.

EMILIANO, P. C. Fundamentos e aplicações dos critérios de informação: akaike e bayesiano. 2009. 92 f. Dissertação (Mestrado em Estatística e Experimentação Agropecuária) - Universidade Federal de Lavras, Lavras, 2009.

ENDERS, W. Applied econometrics time series. 2. ed. New York: Wiley, 2004.

ENGLE, R F.; GRANGER, C. W. J. Co-integration and error correction: Representation, estimation and testing. Econometrica, Chicago, v. 55, n. 2, p. 251-276, 1987.

ENGLE, R. F.; KRONER, K. F. Multivariate Simultaneous Generalized ARCH. Econometric Theory, Cambridge, v. 11, n. 1, p. 122-150, 1995.

FIGLEWSKI, S. Hedging performance and basis risk in stock index futures. The Journal of Finance, San Francisco, v. 39, n. 3, p. 657-669, jul. 1984.

FREITAS, C. A.; ALVES, W. B. Análise da efetividade e da razão ótima do hedge do boi gordo e do cross-hedge do bezerro no estado de São Paulo (2001 a 2010). Análise Econômica, Porto Alegre, ano 31, n. 60, p. 175-202, set. 2013.

GRANGER, C.; NEWBOLD, P. Spurious regressions in econometrics. Journal of Econometrics, Nottingham, v. 2, p. 111-120, jul. 1974.

HARRIS, R. I. D. Using cointegration analysis in econometric modelling. Wheatsheaf: Prentice Hall/ Havester, 1995. 176 p.
HAYENGA, M. L.; DI PIETRE, D. D. Cross-hedging wholesale porks products using live hog futures. American Journal of Agricultural Economics, New York, 1982, v. 64, n. 4, p. 747-751.

INSTITUTO BRASILEIRO DE GEOGRAFIA E ESTATÍSTICA(IBGE). Produção da pecuária municipal: efetivo dos rebanhos no Brasil. 2015. Disponível em: http://www.sidra.ibge.gov.br. Acesso em: 02 dez. 2016.

JOHANSEN, S. Statistical analysis of cointegration vectors. Journal of Economics Dynamics and Control, Frankfurt, v. 12, p. 231-254, 1988.

JOHNSON, L. L. The theory of hedging and speculation in commodity futures. The Review of Economics Studies, Oxford, UK, v. 27, n. 1, p. 139-151, jun. 1960.

LIEN, D.; TSE, Y. K. Some recent developments in futures hedging. Journal of Economic Surveys, Malden, v. 16, n. 1, p. 357-396, 2002.

LORD, Y. S.; TURNER, S. C. Basis risk for rice. In: AMERICAN AGRICULTURAL ECONOMICS ASSOCIATION ANNUAL MEETING, 1998, Milwaukee. Anais... Salt Lake City: AAEA, 1998. p. 1-13.

LUTKEPOHL, H. Introduction to multiple time series analysis. New York: Springer-Verlag. 1991.

MATTOS, F. L.; GARCIA, P. Price discovery and risk transfer in thinly traded markets: evidence from Brazilian agricultural futures markets. Review of Futures Markets, Washington, DC, v. 14, n. 4, p. 471-483, 2006.

MOITA, R. M.; GOLON, L. A. Oligopsônio dos frigoríficos: uma análise empírica de poder de mercado. Revista de Administração Contemporânea, Rio de Janeiro, v. 18, n. 6, p. 1-23, nov./dez. 2014.

MONTEIRO, W. F.; RODRIGUES, M. A.; ALVES, A. F.; PARRÉ, J. L. A não efetividade do hedge para o boi gordo. Revista de Economia, Curitiba, v. 39, n. 1 (ano 37), p. 149-166, jan./abr., 2013.

MYERS, R. J.; THOMPSON, S. R. Generalized optimal hedge ratio estimation. American Journal of Agricultural Economics, New York, v. 71, n. 4, p. 858-868, nov. 1989. 
NETZ, J. S. An empirical test of the effect of basis risk on cash markets positions. Journal of Futures Markets, Malden, v. 16, n. 3, p. 289-311, mai. 1996.

OLIVEIRA NETO, O. J.; REZENDE, S. O.; FIGUEIREDO, R. S. Efetividade das operações de hedge do boi gordo no mercado futuro da BM\&F para o estado de Goiás. Revista de Economia da UEG, Goiânia, v. 5, n. 2, p. 42-69, 2009.

SILVEIRA, R. L. F. Análise das operações de cross hedge do bezerro e do hedge do boi gordo no mercado futuro da BM\&F. 2003. 106 f. Dissertação (Mestrado em Economia Aplicada) - Escola Superior de Agricultura “Luiz de Queiroz", Universidade de São Paulo, Piracicaba, 2003.
URSO, F. S. P.; BARRIONUEVO FILHO, A. Análise do poder de mercado no setor de carne bovina no Brasil: a relação entre pecuaristas e frigoríficos. In: Encontro Nacional de Economia, 36, 2008, Niterói. Anais... Salvador: ANPEC, 2008. p. 1-16.

VISWANATH, P. V. Efficient use of information, convergence adjustments, and regression estimates of hedge ratios. Journal of Futures Markets, Malden, v. 13, n. 1, p. 43-53, 1993.

ZILLI, J. B.; SILVA, A. F.; CAMPOS, S. K.; COSTA, J. S. Análise da cointegração e causalidade dos preços de boi gordo em diferentes praças nas regiões Sudeste e Centro-Oeste do Brasil. Revista de Economia Agrícola, São Paulo, v. 55, n. 2, p. 105-119, jul./dez., 2008. 